\title{
A redescription of data does not count as a general theory
}

\author{
Serge H. Ahmed
}

Received: 16 December 2013 / Accepted: 1 February 2014 / Published online: 27 May 2014

(C) Springer-Verlag Berlin Heidelberg 2014

Piazza and Deroche-Gamonet (PDG) seek to formulate a framework for addiction sufficiently broad to encompass most data in the field and, notably, those produced by the Bordeaux School of Psychobiology to which I also belong. Briefly, according to the PDG framework, the development of addiction would involve two unidirectional transitions across three consecutive stages of drug use: a first transition from recreational drug use to escalated drug use and a second transition from escalated drug use to compulsive drug use. Importantly, the likelihood that a given individual drug user will make a transition would depend on two types of psychobiological vulnerability that are transition-specific and differently malleable to drug availability. Specifically, the vulnerability to transition from recreational to escalated drug use would be different from and more drug-malleable than the vulnerability to transition from escalated to compulsive drug use.

Overall, this is an interesting proposal. However, I would not call it a general theory, but a redescription of existing data. First, PDG framework does not advance any overarching theoretical construct and relies on a shaky theoretical foundation. The authors claim to have derived it from a careful consideration of both DSM-based and RDoC-like behavioral symptoms, but how is this derivation made? What are the theoretical principles and rules involved? How can one deduce a dynamical multistage framework for the transition to addiction from a heterogeneous set of static, operational, and descriptive behavioral criteria? Clearly, something is missing here. Without further explanation, PDG framework appears

S. H. Ahmed ( $\bowtie)$

Université de Bordeaux, Institut des Maladies Neurodégénératives, UMR 5293, 146 rue Léo-Saignat, 33000 Bordeaux, France e-mail: sahmed@u-bordeaux2.fr

S. H. Ahmed

CNRS, Institut des Maladies Neurodégénératives, UMR 5293, 146 rue Léo-Saignat, 33000 Bordeaux, France more as an ad hoc and arbitrary redescription of existing data than a general theory of addiction. It indeed abounds in unjustified claims. For instance, what does forbid the existence of a direct transition from recreational to compulsive drug use without transitioning to the stage of escalated drug use? What does prevent a transition to be bidirectional and reversible instead of being unidirectional? What does exclude a stage of abstinence and associated specific transitions and vulnerabilities? Abstinence necessarily precedes recreational drug use and should eventually follow any other stage of drug use at least in the majority of illegal drug users (Heyman 2013). Second, the explanatory power of this framework is currently uncertain. At a minimum, one expects from a novel framework to be as good as or preferably better than the previous ones in organizing, encompassing, and/or explaining existing data. Whether PDG framework meets this minimum requirement is currently unknown, however, mainly because the authors do not make the effort to compare their work with that of others (e.g., Kendler et al. 2012; Redish et al. 2008). Third, the heuristic value of PDG framework is low as measured by its inability to generate truly novel and unique predictions and/or to help see and understand things differently. I was at pain to formulate or even identify truly novel predictions from PDG framework. The heuristic impotence of PDG framework is not surprising and directly follows from its ad hoc descriptive nature.

A mere redescription of data is not necessarily a fatal flaw and can even sometimes prove to be instrumental in the formulation of a general theory, as illustrated, for instance, by the early history of genetics or quantum mechanics. However, in the case of PDG framework, the data themselves are still open for debate and reinterpretation. First, PDG interprets the classic LR-HR rat model as a model of the transition from recreational to escalated drug use. This is inaccurate. It is true that the LR-HR model was highly influential in the 1990s and spawned a productive line of research on individual factors 
that influence acquisition of low-dose drug self-administration under short access conditions. Ironically, however, by focusing attention on drug use initiation, this model has contributed, perhaps more than any other models, to blur the distinction between mere drug use and addiction and thus retard progress in this area. If anything, the LR-HR model may be considered a model of transition from initial abstinence to recreational drug use, but certainly not a model of transition to escalated drug use (Ahmed 2011; Ahmed and Koob 1998). It remains to be demonstrated whether HR rats are more vulnerable than LR rats to escalation of drug use under extended access conditions. Second, PDG claims to have demonstrated that regardless of drug exposure, only few vulnerable rats would develop cocaine addiction after prolonged drug use while the remaining majority would be resilient. It may be true that only a few rats can develop addiction, but technically, PDG did not discover that this is indeed the case, as already explained elsewhere (Ahmed 2010, 2012). What they did instead was to invent a DSM-like addiction checklist method for rats that produces this outcome. The problem is that this method cannot, by design, produce a different outcome, say, that $60 \%$ of rats develop addiction. Clearly, it presupposes what it pretends to demonstrate and not the other way around. This circular method explains why the rate of rats with addiction-like behavior cannot change much with further extending drug access time. This would also explain why comparable outcomes should be observed if this method was to be applied to other drugs of abuse (e.g., heroin). Finally, PDG insist, rightly so, on defining individuals with addiction as individuals whose behavioral repertoire is predominantly colonized by drug use at the expense of other activities. However, none of their operational criteria of addiction in rats come close to capture such "behavioral colonization." This is mainly because in their studies, like in mainstream studies in the field, rats are given access to drugs for self-administration without other goals or activities (Ahmed 2010; Ahmed et al. 2013). To borrow the water tank metaphor of PDG, one could say that the brain of a rat in the standard drug self-administration setting resembles a water tank with only one floating cylinder corresponding to the "drug use" goal. Clearly, with one cylinder floating, the physical state of water (liquid or solid) in the tank does not matter much. Be it frozen or not, a rat's brain is still stuck with only one goal! One way to resolve this limitation is to enrich the behavioral repertoire of rats with other competing activities during drug access and see whether drug use becomes the preferred or dominant activity at least in some individual rats (Ahmed 2010; Ahmed et al. 2013). However, this will only be a first step towards a more valid animal model of addiction. Another, more difficult step, still unattained, will be to show that this behavior is disordered, that is, it should be caused by a harmful dysfunction in the individual and not by other non-pathological factors (Spitzer and Wakefield 1999; Wakefield 1992).

Acknowledgments This work is supported by the French Research Council (CNRS), the French National Agency (ANR), the Fondation NRJ, the University of Bordeaux, and the Conseil Regional d'Aquitaine. I thank Drs. Heather Madsen and Karine Guillem for their comments on an early draft of this article.

\section{References}

Ahmed SH (2010) Validation crisis in animal models of drug addiction: beyond non-disordered drug use toward drug addiction. Neurosci Biobehav Rev 35:172-184

Ahmed SH (2011) Escalation of drug use. In: Olmstead MC (ed) Animal models of drug addiction, neuromethods, vol 53. Humana, New York, pp 267-292

Ahmed SH (2012) The science of making drug-addicted animals. Neuroscience 211:107-125

Ahmed SH, Koob GF (1998) Transition from moderate to excessive drug intake: change in hedonic set point. Science 282:298-300

Ahmed SH, Lenoir M, Guillem K (2013) Neurobiology of addiction versus drug use driven by lack of choice. Curr Opin Neurobiol 23: $581-587$

Heyman GM (2013) Quitting drugs: quantitative and qualitative features. Annu Rev Clin Psychol 9:29-59

Kendler KS, Chen X, Dick D, Maes H, Gillespie N, Neale MC, Riley B (2012) Recent advances in the genetic epidemiology and molecular genetics of substance use disorders. Nat Neurosci 15:181-189

Redish AD, Jensen S, Johnson A (2008) A unified framework for addiction: vulnerabilities in the decision process. Behav Brain Sci 31:415-437, discussion 437-87

Spitzer RL, Wakefield JC (1999) DSM-IV diagnostic criterion for clinical significance: does it help solve the false positives problem? Am J Psychiatry 156:1856-1864

Wakefield JC (1992) The concept of mental disorder. On the boundary between biological facts and social values. Am Psychol 47:373-388 